\title{
SOME CLOSED-FORM BENDING FORMULAS FOR ELASTICALLY RESTRAINED EULER-BERNOULLI BEAMS UNDER POINT AND UNIFORMLY DISTRIBUTED LOADS
}

\author{
Vebil Yıldırım \\ Department of Mechanical Engineering, University of Çukurova \\ Adana, Turkey \\ vebil@cu.edu.tr
}

Received: 19 July 2018; Accepted: 28 October 2018

\begin{abstract}
The transfer matrix method based on the Euler-Bernoulli beam theory is employed in order to originally achieve some exact analytical formulas for elastically supported beams under a point force together with uniformly distributed force and uniformly distributed couple moments. Those closed-form formulas can be used in a variety of engineering applications especially at the pre-design stage to get an insight into the response of the structure. Contrary to the classical boundary conditions, it is also observed that the Euler-Bernoulli solutions of a beam with elastic supports are sensitive to the ratio of length to thickness $(L / h)$.
\end{abstract}

MSC 2010: $74 K 10,74 B 05,35 C 05,35 G 10$

Keywords: Euler-Bernoulli beam, elastic support, transfer matrix method, initial value problem, bending

\section{Introduction}

As it is well known, the Euler-Bernoulli beam theory (also known as the engineer's beam theory or classical beam theory), which was first introduced circa 1750, still provides a simple calculation tool to analyse numerous static and dynamic engineering problems [1-5]. The underlying well-known assumptions in the Euler-Bernoulli theory are: i) The cross-section is infinitely rigid in its own plane, ii) The cross-section of a beam remains plane after deformation, iii) The plane section initially perpendicular to the mid-surface will remain normal to the deformed axis of the beam. Based on the experimental measurements, these assumptions are held for long and slender beams.

The transfer matrix method also provides the scientists with a simple tool to model and solve one-dimensional problems [6-9]. The simplicity and easy programmability of the transfer matrix method makes it an alternative method to the finite elements in structural and mechanical engineering. 
The present study is a continuation of Ref. [9] in which some exact analytical bending formulas for classically supported Euler-Bernoulli beams under both concentrated and generalized power/sinusoidal distributed loads were offered. As stated in the Abstract, an Euler-Bernoulli beam supported by both linear and rotational springs is to be considered in the present bending analysis.

\section{Application of the Transfer Matrix Method}

Let $x$ be the beam axis, and let's use the prime symbol for the derivative of the related quantity with respect to $x$. The governing homogeneous differential equation set for the out-of-plane bending analysis of the beam having a uniform section in canonical form is given by [6]

$$
\boldsymbol{S}^{\prime}(x)=\boldsymbol{D} \boldsymbol{S}(x), \boldsymbol{S}(x)=\left\{\begin{array}{c}
w(x) \\
\theta(x) \\
M(x) \\
T(x)
\end{array}\right\}, \quad \boldsymbol{D}=\left[\begin{array}{cccc}
0 & -1 & 0 & 0 \\
0 & 0 & \frac{1}{E I} & 0 \\
0 & 0 & 0 & 1 \\
0 & 0 & 0 & 0
\end{array}\right]
$$

where $\boldsymbol{S}(x)$ is called the state vector which comprises the cross-sectional quantities at a positive section, $\boldsymbol{D}$ is the differential matrix, $w(x)$ is the transverse displacement along z-axis, $\theta(x)$ is the rotation about $y$-axis, $M(x)$ is the bending moment, and $T(x)$ is the shear force, and $E I$ is the bending rigidity. Let's denote the unit matrix by $\boldsymbol{J}$ to determine the characteristic equation of the differential matrix as follows

$$
|\boldsymbol{D}-\lambda \boldsymbol{J}|=\lambda^{4}=0
$$

Since every square matrix satisfies its own characteristic equation according to the Cayley-Hamilton theorem, $\boldsymbol{D}^{4}=0$ is held. This means that the higher powers of the differential matrix that are equal or greater than four are identically zero.

Both the state vector and the transfer matrix satisfy the similar type of differential equation as in Eq. (1) [6]:

$$
\boldsymbol{F}^{\prime}(x)=\boldsymbol{D} \boldsymbol{F}(x)
$$

If the elements of $\boldsymbol{D}$ are constants as in Eq. (1), one may get an exact transfer matrix. In this case, the solution of Eq. (3) with the initial conditions, $\boldsymbol{F}(x=0)=\boldsymbol{J}$, gives $\boldsymbol{F}(x)$ as follows:

$$
\boldsymbol{F}(x)=e^{x \boldsymbol{D}}=\boldsymbol{I}+x \boldsymbol{D}+\frac{x^{2}}{2 !} \boldsymbol{D}^{2}+\frac{x^{3}}{3 !} \boldsymbol{D}^{3}=\left[\begin{array}{cccc}
1 & -x & -\frac{x^{2}}{2 E I} & -\frac{x^{3}}{6 E I} \\
0 & 1 & \frac{x}{E I} & \frac{x^{2}}{2 E I} \\
0 & 0 & 1 & x \\
0 & 0 & 0 & 1
\end{array}\right]
$$


Let's assume that the beam is to be subjected to both a distributed force $q(x)$ and a distributed couple moment $\mathrm{m}(x)$ along the beam axis together with a concentrated force $P_{o}$ and a couple moment $\mu_{o}$ acting at section $x=a$. Under this assumption, the overall transfer matrix relates the state vectors at both ends of the beam as follows:

$$
\mathbf{S}(L)=\boldsymbol{F}(L) \boldsymbol{S}(0)+\int_{0}^{L} \boldsymbol{F}(L-\xi) \boldsymbol{k}(\xi) \mathrm{d} \xi+\boldsymbol{F}(L-a) \boldsymbol{K}(a)
$$

where $\boldsymbol{k}(\xi)$ stands for the nonhomogeneous solution due to the distributed forces, and $\boldsymbol{K}(a)$ is referred to as a discontinuity matrix due to the intermediate point loads

$$
\boldsymbol{k}(\xi)=\left\{\begin{array}{c}
0 \\
0 \\
-m(\xi) \\
-q(\xi)
\end{array}\right\}, \quad \boldsymbol{K}(a)=\left\{\begin{array}{c}
0 \\
0 \\
-\mu_{o} \\
-P_{o}
\end{array}\right\}
$$

For short, let's use the following for symbolizing the elements of the state vectors at two ends

$$
\boldsymbol{S}(0)=\left\{\begin{array}{c}
w(0) \\
\theta(0) \\
M(0) \\
T(0)
\end{array}\right\}=\left\{\begin{array}{c}
w_{o} \\
\theta_{o} \\
M_{o} \\
T_{o}
\end{array}\right\}, \quad \boldsymbol{S}(L)=\left\{\begin{array}{c}
w(L) \\
\theta(L) \\
M(L) \\
T(L)
\end{array}\right\}=\left\{\begin{array}{c}
w_{L} \\
\theta_{L} \\
M_{L} \\
T_{L}
\end{array}\right\}
$$

Boundary conditions considered in the present study are shown in Figure 1 and Table 1. In Figure 1 and Table $1, k_{o}$ and $k_{L}$ stand for the linear spring constants at the initial and final ends, respectively. The rotational spring constants at the ends are represented by $m_{o}$ and $m_{L}$.

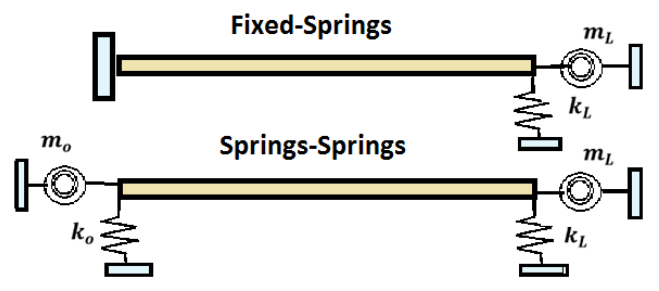

Fig. 1. Elastically supported beams

Boundary conditions considered

Table 1

\begin{tabular}{|l|c|c|}
\hline & $x=0$ & $x=L$ \\
\hline Clamped-Elastic (C-E) & $w_{o}=0, \theta_{o}=0$ & $T_{L}=-k_{L} w_{L}, M_{L}=-m_{L} \theta_{L}$ \\
\hline Elastic-Elastic (E-E) & $T_{o}=k_{o} w_{o}, M_{o}=m_{o} \theta_{o}$ & $T_{L}=-k_{L} w_{L}, M_{L}=-m_{L} \theta_{L}$ \\
\hline
\end{tabular}


In the transfer matrix method, after evaluation of the transfer matrix, it is essential to calculate whole elements the whole elements of the initial state vector as a second key stage of the procedure. To do this, the boundary conditions given at both ends (Table 1) should be implemented into Eq. (5) to form the equations for the unknown quantities at the initial end of the beam. The unknown elements of the initial state vector are then obtained from the solution of the equations generated in this way. After determination of the full elements of $\boldsymbol{S}(0)$, all sectional quantities at any section may be computed in a straight way as follows

$$
\begin{aligned}
& \text { For }(0 \leq x<a), \quad \boldsymbol{S}^{I}(\mathrm{x})=\boldsymbol{F}(x) \boldsymbol{S}(0)+\int_{0}^{x} \boldsymbol{F}(x-\xi) \boldsymbol{k}(\xi) \mathrm{d} \xi \\
& \text { For }(a \leq x \leq L), \boldsymbol{S}^{I I}(\mathrm{x})=\boldsymbol{F}(x) \boldsymbol{S}(0)+\int_{0}^{x} \boldsymbol{F}(x-\xi) \boldsymbol{k}(\xi) \mathrm{d} \xi+\boldsymbol{F}(x-a) \boldsymbol{K}(a)
\end{aligned}
$$

In the following two sections, the analytical formulas are to be derived for beams under separate distributed and concentrated loads. Since small deformations are assumed, the superposition principle is held when necessary.

\section{Solutions for uniformly distributed forces}

If only uniformly distributed forces and couple moments are concerned, $q(x)=-q_{o}$ and $m(x)=-m_{d}$, a general solution takes the following form $(0 \leq x \leq L)$

$$
\mathbf{S}(\mathrm{x})=\boldsymbol{F}(x) \boldsymbol{S}(0)+\int_{0}^{x} \boldsymbol{F}(x-\xi) \boldsymbol{k}(\xi) \mathrm{d} \xi=\boldsymbol{F}(x) \boldsymbol{S}(0)+\left\{\begin{array}{c}
\frac{x^{3}\left(4 m_{d}+x q_{o}\right)}{24 \mathrm{EI}} \\
-\frac{x^{2}\left(3 m_{d}+x q_{o}\right)}{6 \mathrm{EI}} \\
-\frac{1}{2} x\left(2 m_{d}+x q_{o}\right) \\
-x q_{o}
\end{array}\right\}
$$

\subsection{A C-E beam under uniformly distributed loads}

In a C-E beam, the unknown elements of the initial state vector are found as follows after implementation of the boundary conditions as (Table 1)

$$
M_{o}=\frac{L\left(72 \mathrm{EI}_{d}\left(2 \mathrm{EI}+L m_{L}\right)-L q_{o}\left(\begin{array}{c}
L^{3} k_{L}\left(6 \mathrm{EI}+L m_{L}\right) \\
+24 \mathrm{EI}\left(3 \mathrm{EI}+2 L m_{L}\right)
\end{array}\right)\right)}{12 L^{3} k_{L}\left(4 \mathrm{EI}+L m_{L}\right)+144 \mathrm{EI}\left(\mathrm{EI}+L m_{L}\right)}
$$




$$
T_{o}=\frac{L\left(L^{2} k_{L}\left(\begin{array}{c}
2 m_{d}\left(4 \mathrm{EI}+L m_{L}\right) \\
+L q_{o}\left(5 \mathrm{EI}+L m_{L}\right)
\end{array}\right)+24 \mathrm{EI} q_{o}\left(\mathrm{EI}+L m_{L}\right)\right)}{2 L^{3} k_{L}\left(4 \mathrm{EI}+L m_{L}\right)+24 \mathrm{EI}\left(\mathrm{EI}+L m_{L}\right)}
$$

Cross-sectional quantities are then derived in closed forms as

$$
\boldsymbol{S}_{C-E}^{E}(\mathrm{x})=\left\{\begin{array}{c}
\frac{L^{3}\left(L q_{o}\left(3 \mathrm{EI}+L m_{L}\right)-2 m_{d}\left(4 \mathrm{EI}+L m_{L}\right)\right)}{2 L^{3} k_{L}\left(4 \mathrm{EI}+L m_{L}\right)+24 \mathrm{EI}\left(\mathrm{EI}+L m_{L}\right)} \\
\frac{L^{2}\left(72 \mathrm{EI} m_{d}+L q_{o}\left(L^{3} k_{L}-24 \mathrm{EI}\right)\right)}{12 L^{3} k_{L}\left(4 \mathrm{EI}+L m_{L}\right)+144 \mathrm{EI}\left(\mathrm{EI}+L m_{L}\right)} \\
-\frac{L^{2} m_{L}\left(72 \mathrm{EI} m_{d}+L q_{o}\left(L^{3} k_{L}-24 \mathrm{EI}\right)\right)}{12\left(L^{3} k_{L}\left(4 \mathrm{EI}+L m_{L}\right)+12 \mathrm{EI}\left(\mathrm{EI}+L m_{L}\right)\right)} \\
\frac{L^{3} k_{L}\left(2 m_{d}\left(4 \mathrm{EI}+L m_{L}\right)-L q_{o}\left(3 \mathrm{EI}+L m_{L}\right)\right)}{2 L^{3} k_{L}\left(4 \mathrm{EI}+L m_{L}\right)+24 \mathrm{EI}\left(\mathrm{EI}+L m_{L}\right)}
\end{array}\right\}
$$

Dimensionless transverse displacements under uniform loads are defined as $\bar{w}=\left(E I /\left(q_{o} L^{4}\right)\right) w$. As a numerical example, two different spring constants are studied. They are chosen as $m_{L}=1.10^{4} \mathrm{Nm} / \mathrm{rad}, k_{L}=1.10^{4} \mathrm{~N} / \mathrm{m}$ and $m_{L}=1.10^{6} \mathrm{Nm} / \mathrm{rad}, k_{L}=1.10^{6} \mathrm{~N} / \mathrm{m}$. The properties of the beam with a square section are: $\quad L=1 \mathrm{~m}=$ constant, $q_{o}=1 \mathrm{~N} / \mathrm{m}, E=30 \mathrm{MPa}, I=h^{4} / 12$. Dimensionless transverse displacements in a $\mathrm{C}$-E beam based on the two beam theories are shown in Figure 2 and Table 2. For $L / h=10$, the Timoshenko solution gives higher transverse dimensionless displacements for all soft and stiff elastic springs.
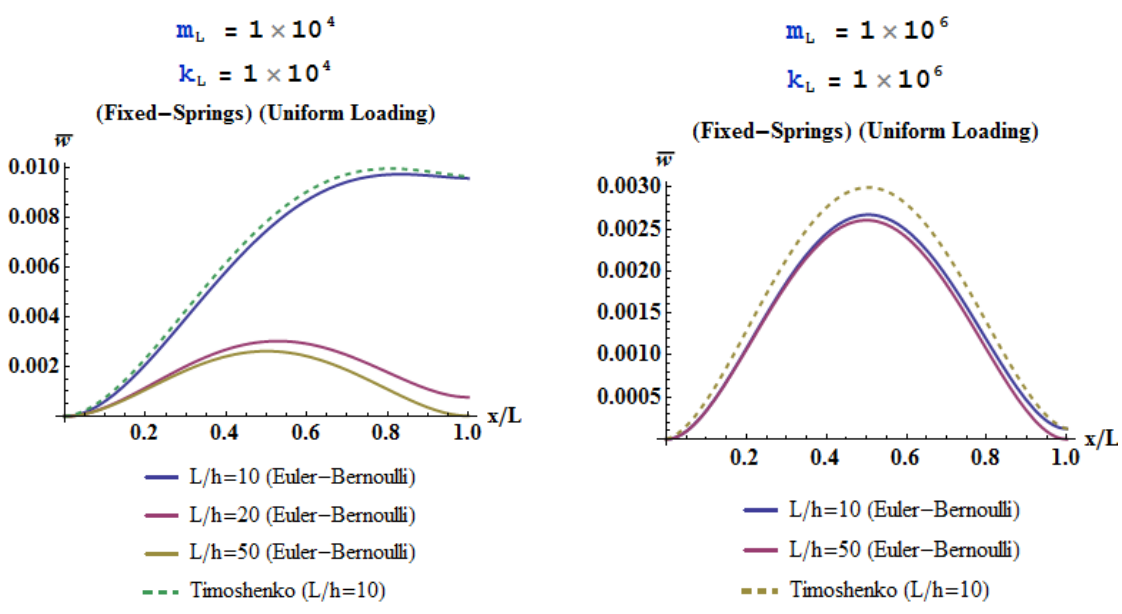

Fig. 2. Dimensionless transverse displacements in a C-E beam based on the two beam theories 
Table 2

Transverse displacements in C-E and E-E beams under uniformly distributed forces

\begin{tabular}{|c|c|c|c|c|c|}
\hline \multirow{3}{*}{$x / L$} & $L / h=10$ & $L / h=20$ & $L / h=50$ & $L / h=10$ & $L / h=20$ \\
\hline & \multicolumn{5}{|c|}{$m_{L}=1.10^{6} ; k_{L}=1.10^{6}$} \\
\hline & \multicolumn{3}{|c|}{ C-E Timoshenko } & \multicolumn{2}{|c|}{ E-E Timoshenko } \\
\hline 0. & 0. & 0. & 0. & 0.000125 & $7.8125 .10^{-6}$ \\
\hline 0.2 & 0.00128868 & 0.00111953 & 0.00107501 & 0.001403 & 0.00112669 \\
\hline 0.6 & 0.00279552 & 0.00248325 & 0.00241261 & 0.002842 & 0.00248612 \\
\hline \multirow[t]{2}{*}{1.} & 0.000124607 & $7.81093 .10^{-6}$ & $1.99999 .10^{-7}$ & 0.000125 & $7.8125 .10^{-6}$ \\
\hline & \multicolumn{3}{|c|}{ C-E Euler-Bernoulli } & \multicolumn{2}{|c|}{ E-E Euler-Bernoulli } \\
\hline 0. & 0. & 0 & 0. & 0.000125 & $7.8125 .10^{-6}$ \\
\hline 0.2 & 0.00108028 & 0.00106752 & 0.00106669 & 0.001195 & 0.00107469 \\
\hline 0.6 & 0.00248371 & 0.00240525 & 0.00240013 & 0.00253 & 0.00240812 \\
\hline \multirow[t]{3}{*}{1.} & 0.000124595 & $7.81091 .10^{-6}$ & $1.99999 .10^{-7}$ & 0.000125 & $7.8125 .10^{-6}$ \\
\hline & \multicolumn{5}{|c|}{$m_{L}=1.10^{4} ; k_{L}=1.10^{4}$} \\
\hline & \multicolumn{3}{|c|}{ C-E Timoshenko } & \multicolumn{2}{|c|}{ E-E Timoshenko } \\
\hline 0. & 0. & 0. & 0. & 0.0125 & 0.00078125 \\
\hline 0.2 & 0.00232318 & 0.00120285 & 0.00107717 & 0.0140921 & 0.00192069 \\
\hline 0.6 & 0.00901646 & 0.00299159 & 0.00242591 & 0.0156882 & 0.0032904 \\
\hline \multirow[t]{2}{*}{1.} & 0.00961267 & 0.000765863 & 0.0000199896 & 0.0125 & 0.00078125 \\
\hline & \multicolumn{3}{|c|}{ C-E Euler-Bernoulli } & \multicolumn{2}{|c|}{ E-E Euler-Bernoulli } \\
\hline 0. & 0. & 0 & 0. & 0.0125 & 0.00078125 \\
\hline 0.2 & 0.00207851 & 0.00115022 & 0.00106885 & 0.0138841 & 0.00186869 \\
\hline 0.6 & 0.00867176 & 0.00291381 & 0.00241343 & 0.0153762 & 0.0032124 \\
\hline 1. & 0.00954707 & 0.000765746 & 0.0000199896 & 0.0125 & 0.00078125 \\
\hline
\end{tabular}

Contrary to the classical supports, Euler-Bernoulli solutions of beams with elastic supports display sensitivity to the ratio of $L / h$. Develops with using much softer springs (Consider revising here. Oddly-worded and not sure of intent). Soft springs make the transverse dimensionless displacements higher at the elastically supported ends. Those displacements decrease with increasing ratios of $L / h$ for the same beam. As it is well known, when both spring constants get larger and larger, viz., when $m_{L} \rightarrow \infty$ and $k_{L} \rightarrow \infty$, the elastic supports turn to be rigid ones in the limit case.

\subsection{An E-E beam under uniformly distributed loads}

The beam is, now, assumed to be elastically supported at two ends by using both the linear and rotational springs (Fig. 1). The elements of $\boldsymbol{S}(0)$ are as follows 
$w_{o}=\frac{L\left(L k_{L}\left(\begin{array}{c}2 m_{d}\left(\begin{array}{c}L m_{o}\left(4 E I+L m_{L}\right) \\ +4 E I\left(3 E I+L m_{L}\right)\end{array}\right) \\ +L q_{o}\left(\begin{array}{c}L m_{o}\left(5 E I+L m_{L}\right) \\ +3 E I\left(4 E I+L m_{L}\right)\end{array}\right)\end{array}\right)+24 E I q_{o}\left(m_{L}\left(E I+L m_{o}\right)+E I m_{o}\right)\right.}{2 k_{L}\left(\begin{array}{c}L^{2} k_{o}\left(\begin{array}{c}L m_{o}\left(4 E I+L m_{L}\right) \\ +4 E I\left(3 E I+L m_{L}\right)\end{array}\right) \\ +12 E I\left(m_{o}\left(E I+L m_{L}\right)+E I m_{L}\right)\end{array}\right)+24 E I k_{o}\left(m_{L}\left(E I+L m_{o}\right)+E I m_{o}\right)}$

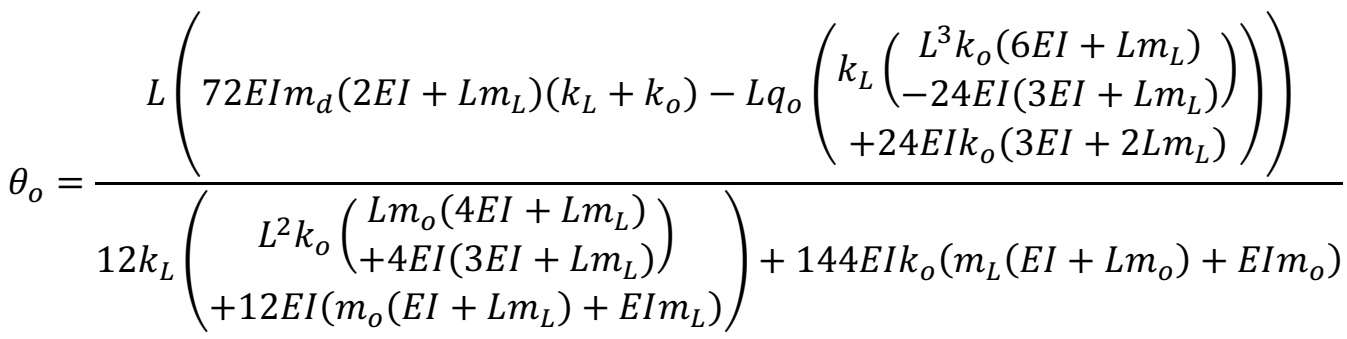

$$
\begin{aligned}
& T_{o}=k_{o} w_{o}, \quad M_{o}=m_{o} \theta_{o}
\end{aligned}
$$

Sectional quantities along the beam are explicitly presented in Eq. (13):

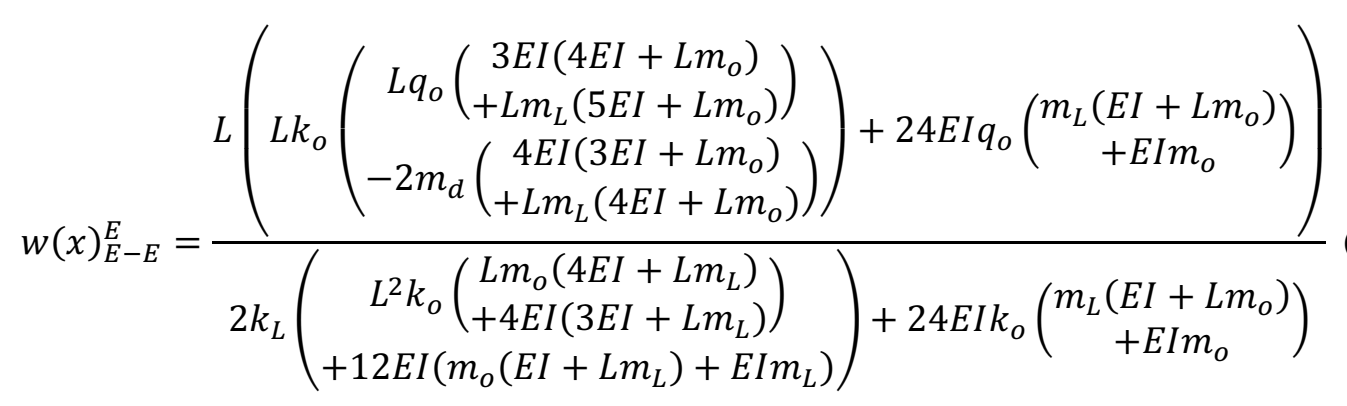

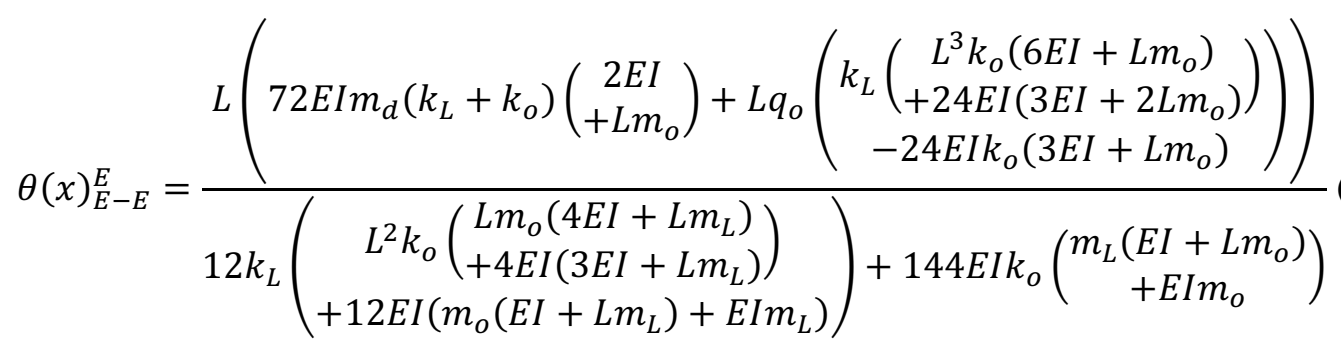




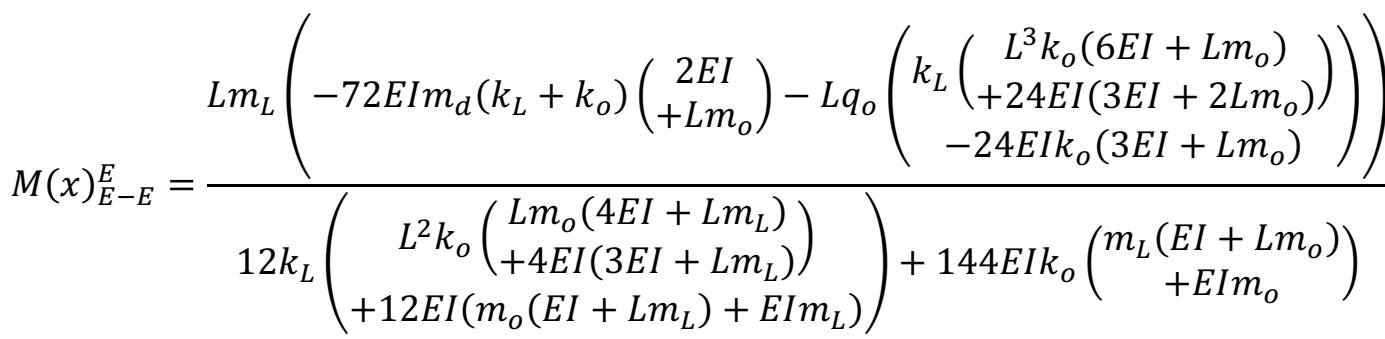

$T(x)_{E-E}^{E}=-\frac{L k_{L}\left(L k_{o}\left(\begin{array}{c}L q_{o}\left(\begin{array}{c}3 E I\left(4 E I+L m_{o}\right) \\ +L m_{L}\left(5 E I+L m_{o}\right)\end{array}\right) \\ -2 m_{d}\left(\begin{array}{c}4 E I\left(3 E I+L m_{o}\right) \\ +L m_{L}\left(4 E I+L m_{o}\right)\end{array}\right)\end{array}\right)+24 E I q_{o}\left(\begin{array}{c}\left.m_{L}\left(\begin{array}{c}E I \\ +L m_{o}\end{array}\right)\right) \\ +E I m_{o}\end{array}\right)\right)}{2 k_{L}\left(\begin{array}{c}L^{2} k_{o}\left(\begin{array}{c}L m_{o}\left(4 E I+L m_{L}\right) \\ +4 E I\left(3 E I+L m_{L}\right)\end{array}\right) \\ +12 E I\left(m_{o}\left(E I+L m_{L}\right)+E I m_{L}\right)\end{array}\right)+24 E I k_{o}\left(\begin{array}{c}m_{L}\left(E I+L m_{o}\right) \\ +E I m_{o}\end{array}\right)}$

A variation of the dimensionless transverse displacement in an E-E beam is demonstrated in Figure 3 for two different spring constants that have been studied in the previous example. Some values of the transverse displacements in an E-E beam based on the two beam theories are also given in Table 2. From Figure 3, a symmetric variation of the transverse displacement is observed due to the symmetric loads and geometry. It is observed from both Table 2 and Figure 3 that an increase in the spring constants results in a decrease in the transverse displacements. In other words, the softer the springs, the greater the dimensionless displacements at both ends. The differences in the dimensionless displacements between the two theories get smaller with softer springs. As $L / h$ increases, the transverse dimensionless displacements reduce. As a conclusion, it is again revealed that Euler-Bernoulli beams supported by elastic springs at both of its ends are sensitive to the variation of $L / h$ ratios for especially soft springs.
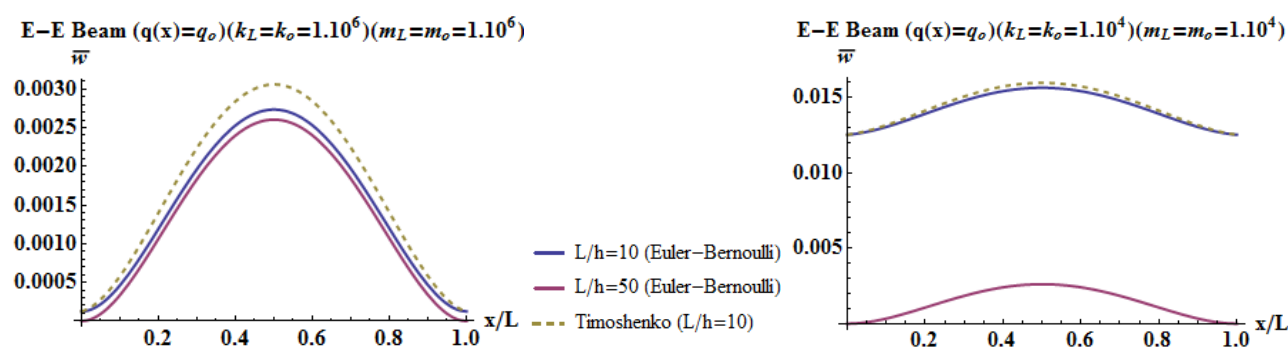

Fig. 3. Deflections in an E-E beam based on the two beam theories 


\section{Solutions for a point force}

In this section, due to the space limitations, beams are assumed to be subjected to only a concentrated force $P_{o}$ acting at $x=a, K(a)=\left\{\begin{array}{llll}0 & 0 & 0 & -P_{o}\end{array}\right\}^{T}$. Under this assumption, the general solution is written before and after $x=a$ as follows

$\boldsymbol{S}(x)^{I}=\boldsymbol{F}(x) \boldsymbol{S}(0), \quad 0 \leq x<a$

$\boldsymbol{S}(x)^{I I}=\left(\begin{array}{c}\boldsymbol{F}(x) \boldsymbol{S}(0) \\ +\boldsymbol{F}(x-a) \boldsymbol{K}(a)\end{array}\right)=\boldsymbol{F}(x) \boldsymbol{S}(0)+\left\{\begin{array}{c}\frac{(a-x)^{2}(x-a) P_{o}}{6 E I} \\ \frac{(a-x)(x-a) P_{o}}{2 E I} \\ (a-x) P_{o} \\ -P_{o}\end{array}\right\}, x \geq a$

The unknown elements of $\boldsymbol{S}(0)$ of a C-E Euler-Bernoulli beam are to be

$$
\begin{gathered}
M_{o}=-\frac{a P_{o}\left(L(a-L) k_{L}\left(\begin{array}{c}
2 \mathrm{EI}(a-2 L) \\
+L(a-L) m_{L}
\end{array}\right)+6 \mathrm{EI}\left(2 \mathrm{EI}-(a-2 L) m_{L}\right)\right)}{L^{3} k_{L}\left(4 \mathrm{EI}+L m_{L}\right)+12 \mathrm{EI}\left(\mathrm{EI}+L m_{L}\right)} \\
T_{o}=\frac{P_{o}\left(k_{L}\left(\begin{array}{c}
2 \mathrm{EI}\left(a^{3}-3 a^{2} L+2 L^{3}\right) \\
+L(2 a+L)(a-L)^{2} m_{L}
\end{array}\right)+12 \mathrm{EI}\left(\mathrm{EI}+L m_{L}\right)\right)}{L^{3} k_{L}\left(4 \mathrm{EI}+L m_{L}\right)+12 \mathrm{EI}\left(\mathrm{EI}+L m_{L}\right)}
\end{gathered}
$$

The unknown elements of the initial state vector of an E-E Euler-Bernoulli beam are achieved as follows $\left(M_{o}=m_{o} \theta_{o}, T_{o}=k_{o} w_{o}\right)$

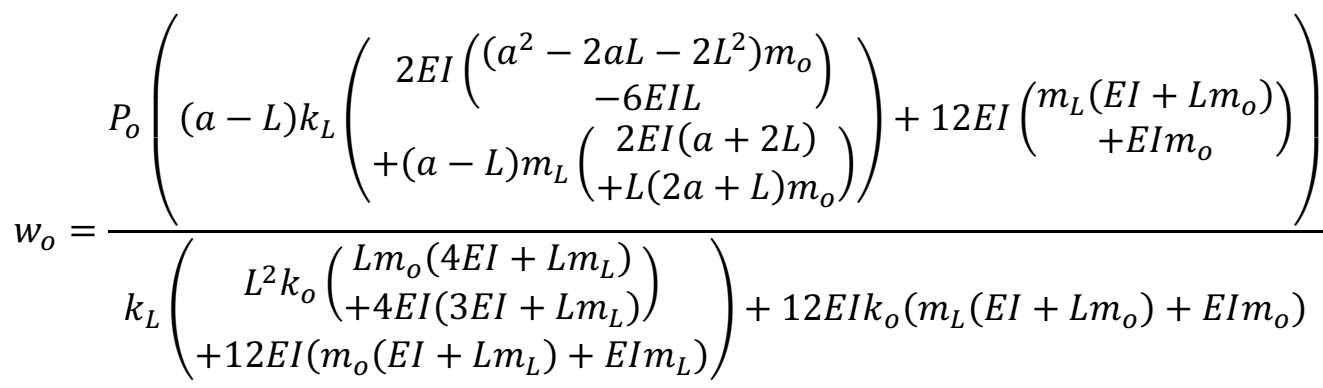

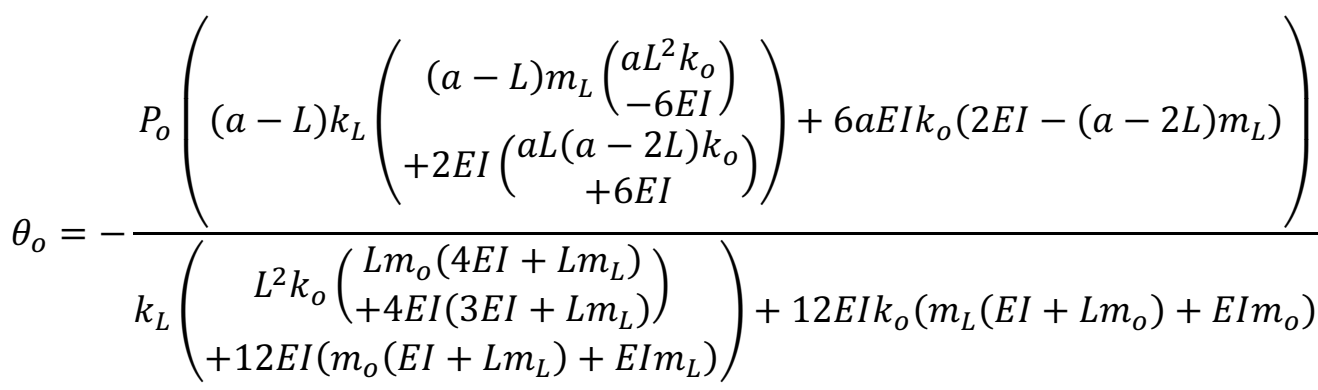


With the help of Eqs. (14)-(16), cross-sectional quantities at any section may be obtained.

\section{Verifications of the results}

Ghannadias and Golmogany [5] presented a sextic B-spline method for the numerical bending solution of a Euler-Bernoulli beam (We use article "a" here because Euler is pronounced "yuler" I do believe) with arbitrary boundary conditions on an elastic Winkler foundation. For an E-E beam under a uniformly distributed force, the following properties are used in this work $\left(1 \mathrm{~kg}_{\mathrm{f}}=9.81 \mathrm{~N}\right)$ [5]: $E=2038901.91 \mathrm{~kg} / \mathrm{cm}^{2}, I=6572.4175 \mathrm{~cm}^{4}, \quad E I=1.340051459406742 \times$ $10^{10}, q_{o}=15 \mathrm{~kg} / \mathrm{cm}, L=500 \mathrm{~cm}, m_{o}=m_{L}=0, k_{o}=k_{L}=2500 \mathrm{~kg} / \mathrm{cm}$. Results are presented in Tables 3 and 4. From those tables, a perfect harmonization is observed between the results.

Table 3

Validation of the present results of an E-E beam under uniformly distributed forces

\begin{tabular}{|c|c|c|c|c|c|c|}
\hline & {$[5] /$ Analitik } & {$[5] /$ B.Spline } & Present & {$[5] /$ Analitik } & {$[5] /$ B.Spline } & Present \\
\hline $\mathrm{x}[\mathrm{cm}]$ & \multicolumn{3}{|c|}{ Displacement [cm] } & \multicolumn{3}{c|}{ Slope [rad] } \\
\hline 0. & 1.5 & 1.5 & 1.5 & -0.00583 & -0.00583 & -0.00583 \\
\hline 50. & 1.78596 & 1.78596 & 1.78596 & -0.00550352 & -0.00550352 & -0.00550352 \\
\hline 100. & 2.04102 & 2.04102 & 2.04102 & -0.00461736 & -0.00461736 & -0.00461736 \\
\hline 150. & 2.2407 & 2.2407 & 2.2407 & -0.00331144 & -0.00331144 & -0.00331144 \\
\hline 200. & 2.3675 & 2.3675 & 2.3675 & -0.00172568 & -0.00172568 & -0.00172568 \\
\hline 250. & 2.41093 & 2.41094 & 2.41094 & 0. & 0. & 0. \\
\hline 300. & 2.3675 & 2.3675 & 2.3675 & 0.00172568 & 0.00172568 & 0.00172568 \\
\hline 500. & 1.5 & 1.5 & 1.5 & 0.00583 & 0.00583 & 0.00583 \\
\hline $\mathrm{x}[\mathrm{cm}]$ & \multicolumn{1}{|c|}{ Bending moment [kgcm] } & & Shear force $[\mathrm{kg}]$ & \\
\hline 0. & 0. & 0. & 0. & 3750. & 3750. & 3750. \\
\hline 50. & 168750. & 168750. & 168750. & 3000. & 3000. & 3000. \\
\hline 100. & 300000. & 300000. & 300000. & 2250. & 2250. & 2250. \\
\hline 150. & 393750. & 393750. & 393750. & 1500. & 1500. & 1500. \\
\hline 200. & 450000. & 450000. & 450000. & 750. & 750. & 750. \\
\hline 250. & 468750. & 468750. & 468750. & 0. & 0. & 0. \\
\hline 300. & 450000. & 450000. & 450000. & -750. & -750. & -750. \\
\hline 500. & 0. & 0. & 0. & -3750. & -3750. & -3750. \\
\hline
\end{tabular}


Table 4

Variation of the maximum displacement $(\mathrm{cm})$ in an E-E beam with the length of the beam

\begin{tabular}{|l|c|c|c|c|}
\hline \multirow{2}{*}{} & \multicolumn{4}{|c|}{$L[\mathrm{~cm}]$} \\
\cline { 2 - 5 } & 100 & 250 & 500 & 1000 \\
\hline Present & 0.301457 & 0.806934 & 2.41094 & 17.575 \\
\hline B. spline [5] & - & - & 2.41094 & - \\
\hline
\end{tabular}

Let us show that the elastic supports return to the rigid ones when very stiff springs are used. The maximum dimensionless displacement in a $\mathrm{C}$-E beam occurs at the mid-span when very stiff springs are used (Table 5). Based on the Euler-Bernoulli beam theory, the maximum dimensionless displacement is evaluated as $\bar{w}_{\text {max }}^{E}=0.00260417$ being insensitive to the variation of the ratio of $L / h$ as seen from Table 5. This value is also equal to the maximum dimensionless displacement in a $\mathrm{C}-\mathrm{C}$ beam. The reason is that very stiff springs may behave as rigid supports.

$$
\left(w_{\max }^{E}\right)_{C-C}=w_{L / 2}^{E}=\frac{q_{o} L^{4}}{384 \mathrm{EI}}=0.00260417 \frac{q_{o} L^{4}}{\mathrm{EI}}=\bar{w}_{L / 2}^{E} \frac{q_{o} L^{4}}{\mathrm{EI}}
$$

Table 5

The maximum displacement in a $\mathrm{C}$-E beam with very stiff springs under uniform forces

\begin{tabular}{|l|c|c|c|c|}
\hline & $L / h=10$ & $L / h=20$ & $L / h=50$ & $L / h=100$ \\
\hline & \multicolumn{4}{|c|}{$\bar{w}_{L / 2}^{E}\left(m_{L}=1 \cdot 10^{24} ; k_{L}=1 \cdot 10^{24}\right)$} \\
\hline Timoshenko & 0.00292917 & 0.00268542 & 0.00261717 & 0.00260742 \\
\hline Euler-Bernoulli & 0.00260417 & 0.00260417 & 0.00260417 & 0.00260417 \\
\hline
\end{tabular}

Some other numerical displacements of this example in both dimensional and dimensionless form are also presented in Table 6 to provide insight into the problem. From this table, it is understood that the dimensionless displacements in a C-E Euler-Bernoulli beam with highly stiff springs, viz., in a C-C Euler-Bernoulli beam are insensitive to the ratio of length to the thickness. This does not mean that Euler-Bernoulli beam dimensional results do not change with the length of the beam.

Table 7 shows the dimensional transverse displacements in a C-E beam with soft springs. This table states that an Euler Bernoulli-beam with elastic supports may be sensitive to the $L / h$ ratios contrary to the classical supports. As expected, dimensional displacements increase with increasing $L / h$ ratios. From Table 2, however, their dimensionless counterparts decrease with increasing $L / h$ ratios. 
Table 6

Both the dimensional and dimensionless displacements in a C-E beam with very stiff springs

\begin{tabular}{|c|c|c|c|c|}
\hline & $L / h=10$ & $L / h=20$ & $L / h=50$ & $L / h=100$ \\
\hline$x / L$ & \multicolumn{2}{|c|}{ DIMENSIONLESS DISPLACEMENT, $m_{L}=1 \cdot 10^{24} ; k_{L}=1 \cdot 10^{24}$, Timoshenko } \\
\hline 0.2 & 0.00127467 & 0.00111867 & 0.00107499 & 0.00106875 \\
0.6 & 0.002712 & 0.002478 & 0.00241248 & 0.00240312 \\
1. & $1.25 \cdot 10^{-22}$ & $7.8125 \cdot 10^{-24}$ & $2 \cdot 10^{-25}$ & $1.25 \cdot 10^{-26}$ \\
\hline \multicolumn{6}{|c|}{ DIMENSIONLESS DISPLACEMENT, $m_{L}=1 \cdot 10^{24} ; k_{L}=1 \cdot 10^{24}$, Euler } \\
\hline 0.2 & 0.00106667 & 0.00106667 & 0.00106667 & 0.00106667 \\
0.6 & 0.0024 & 0.0024 & 0.0024 & 0.0024 \\
1. & $1.25 \cdot 10^{-22}$ & $7.8125 \cdot 10^{-24}$ & $2 \cdot 10^{-25}$ & $1.25 \cdot 10^{-26}$ \\
\hline \multicolumn{5}{|c|}{ DIMENSIONAL DISPLACEMENT (m), $m_{L}=1 \cdot 10^{24} ; k_{L}=1 \cdot 10^{24}$, Timoshenko } \\
\hline 0.2 & $5.09867 \cdot 10^{-6}$ & 0.0000715947 & 0.00268747 & 0.0427499 \\
0.6 & 0.000010848 & 0.000158592 & 0.0060312 & 0.0961248 \\
1. & $5 \cdot 10^{-25}$ & $5 \cdot 10^{-25}$ & $5 \cdot 10^{-25}$ & $5 \cdot 10^{-25}$ \\
\hline \multicolumn{5}{|c|}{ DIMENSIONAL DISPLACEMENT (m), $m_{L}=1 \cdot 10^{24} ; k_{L}=1 \cdot 10^{24}$, Euler } \\
\hline 0.2 & $4.26667 \cdot 10^{-6}$ & 0.0000682667 & 0.00266667 & 0.0426667 \\
0.6 & $9.6 \cdot 10^{-6}$ & 0.0001536 & 5.006 & 0.096 \\
1. & $5 \cdot 10^{-25}$ & $5 \cdot 10^{-25}$ & $5 \cdot 10^{-25}$ & $5 \cdot 10^{-25}$ \\
\hline
\end{tabular}

Table 7

The dimensional displacements in a C-E beam with soft springs

\begin{tabular}{|c|c|c|c|c|}
\hline & $L / h=10$ & $L / h=20$ & $L / h=50$ & $L / h=100$ \\
\hline$x / L$ & \multicolumn{3}{|c|}{ DIMENSIONAL DISPLACEMENT $(\mathrm{m}), m_{L}=1 \cdot 10^{24} ; k_{L}=1 \cdot 10^{24}$, Timoshenko } \\
\hline 0.2 & $9.29273 \cdot 10^{-6}$ & 0.0000769821 & 0.00269294 & 0.0427553 \\
0.6 & 0.0000360659 & 0.000191462 & 0.00606478 & 0.0961584 \\
1. & 0.0000384507 & 0.0000490153 & 0.000049974 & 0.0000499984 \\
\hline \multicolumn{5}{|c|}{ DIMENSIONAL DISPLACEMENT (m), $m_{L}=1 \cdot 10^{24} ; k_{L}=1 \cdot 10^{24}$, Euler } \\
\hline 0.2 & $8.31403 \cdot 10^{-6}$ & 0.0000736139 & 0.00267213 & 0.0426721 \\
0.6 & 0.000034687 & 0.000186484 & 0.00603358 & 0.0960336 \\
1. & 0.0000381883 & 0.0000490077 & 0.000049974 & 0.0000499984 \\
\hline
\end{tabular}

\section{Conclusions}

In the present study, some remarkable formulas were originally proposed for the bending behaviour of elastically supported Euler-Bernoulli beams under both uniformly distributed and concentrated loads via the transfer matrix approach. Dimensional and dimensionless results given in both tabular and graphical forms 
were discussed. It is mainly observed that Euler-Bernoulli beam solutions become sensitive to $L / h$ ratios when they are elastically if it is elastically supported. The author hopes that these formulas will be quite useful when trying to validate purely computational solutions.

\section{References}

[1] Wang, C. (1995). Timoshenko beam-bending solutions in terms of Euler-Bernoulli solutions. Journal of Engineering Mechanics, 121(6), 763-765.

[2] Young, W.C. \& Budynas, R.G. (2002). Roark's Formulas for Stress and Strain, Seventh Edition, McGraw-Hill, New York, ISBN 0-07-072542-X.

[3] Mohammadi, R. (2014). Sextic B-spline collocation method for solving Euler-Bernoulli beam models. Applied Mathematics and Computation, 241, 151-166.

[4] Zamorska, I. (2014). Solution of differential equation for the Euler-Bernoulli beam. Journal of Applied Mathematics and Computational Mechanics, 13(4), 157-162.

[5] Ghannadiasl, A, \& Golmogany, M.Z. (2017). Analysis of Euler-Bernoulli Beams with arbitrary boundary conditions on Winkler foundation using a B-spline collocation method. Engng. Trans., 65(3), 423-445.

[6] İnan, M. (1968). The Method of Initial Values and the Carry-over Matrix in Elastomechanics. ODTÜ Publication, Ankara, No: 20.

[7] Arici, M., \& Granata, M.F. (2011). Generalized curved beam on elastic foundation solved by transfer matrix method. Structural Engineering \& Mechanics, 40(2), 279-295.

[8] Wimmer, H., \& Nachbagauer, K. (2018). Exact transfer- and stiffness matrix for the composite beam-column with Refined Zigzag kinematics. Composite Structures, 189, 700-706.

[9] Yıldırım, V. (2018). Several stress resultant and deflection formulas for Euler-Bernoulli beams under concentrated and generalized power/sinusoidal distributed loads. International Journal of Engineering \& Applied Sciences (IJEAS), 10(2), 35-63. DOI: 10.24107/ijeas. 430666. 